\title{
Thrombophilias and the risk of recurring pregnancy loss in a Mexican population
}

\begin{abstract}
Background: Recurrent gestational loss (RPL) is defined by the ESHRE as the loss of 2 or more consecutive pregnancies. The objective of this study is to evaluate the relationship of Factor V Leiden (FVL, G1691A), prothrombin G20210A (PRT, G20210A), methylenetetrahydrofolate reductase G677A (MTHFR C677AT) and plasminogen activator inhibitor-1 (4G/5G) (PAI-1, 4G/5G); with recurrent gestational loss and perinatal data of Mexican women.

Material and method: Retrospective, observational and cross-sectional study, which includes 277 pregnancies of 95 women and three groups were formed: 1) Control: deliveries of patients without pregnancy loss, without problems during the development of pregnancy and with a study of hereditary thrombophilias, 2) idiopathic fetal death : Deliveries of patients with idiopathic gestational loss $(=1)$ and with study of thrombophilias, and 3) recurrent pregnancy loss. Deliveries of patients with idiopathic recurrent pregnancy loss and with study of hereditary thrombophilias; patient data was collected; age, weight and height, newborn data, weeks of gestation, weight and height, which are reported with mean \pm standard error and analyzed with the student's $t$ test, and thrombophilias, cesarean sections, deliveries and spontaneous abortions are reported in percentages and analyzed with chi2, in both cases the SPSS version 25 statistical package was used.

Results: Of the 95 women included there were no significant differences in age, weight and height in the different rates of each group; one of the thrombophilias to be evaluated in the different populations, it was observed that FVL-G1691A only occurs in recurrent pregnancy loss (15.4\%); the translation of homozygous and heterozygous, it was observed that FVL-G1691A only appeared in recurrent pregnancy loss, perinatal data showed a decrease in the weeks of gestation in newborns of mothers with recurrent pregnancy loss, with a decrease in weight and size.
\end{abstract}

Conclusions: the presence of inherited maternal thrombophilias increases the risk of recurrent pregnancy loss, premature birth, and decreased weight and height at birth.
Volume II Issue 6 - 2020

\author{
Luján Irastorza Jesús Estuardo,' Durand \\ Montaño Carlos,' Kava Braverman \\ Alejandro,' Hernández Ramos Roberto,' \\ Ávila Pérez Felipe de Jesús,' Guerrero Vargas \\ Jose Juan,' Ávila Rebollar Daniela,' Pariente \\ Fernández Maruxa,' Paredes Nuñez María \\ Angélica, ' Vargas Hernández Víctor Manuel ${ }^{2}$ \\ 'Pronatal Clinic (Bité Médica Hospital), México \\ ${ }^{2}$ Female Health Clinic, Mexico
}

Correspondence: Vargas Hernández Víctor Manuel, Female Health Clinic, Mexico, Email vvargashernndez@yahoo.com.mx

Received: October 19, 2020 | Published: November 17, 2020

Keywords: thrombophilias, fetal death, recurrent loss of pregnancy

\section{Background}

Recurrent pregnancy loss is a real disappointment for married couples. Unfortunately, in many cases the exact underlying pathogenesis of Recurrent pregnancy loss remains undetermined, ${ }^{1}$ affecting 1 to $3 \%$ of all couples of reproductive age, ${ }^{2}$ spontaneous abortion is defined as the loss of a fetus at any time between conception and the 24th week of gestation or the loss of a fetus weighing $<500 \mathrm{~g}^{3-5}$ The World Health Organization (WHO) defines recurrent spontaneous abortion as the presence of three or more consecutive spontaneous abortions before 20 weeks of gestation ${ }^{5}$ and the American Society for Reproductive Medicine (ASRM) defines it as the occurrence of two consecutive spontaneous abortions as recurrent abortion; ; 3 this definition increases the incidence of recurrent abortion to $5 \%$ of all couples of reproductive age; ${ }^{7}$ The same definition as the European Society for Human Reproduction and Embryology (ESHRE) will be the basis for this study. ${ }^{8}$ There is consensus that women should not undergo a comprehensive evaluation after a single pregnancy loss in the first trimester or at the beginning of the second trimester, they are common and sporadic events with a slightly higher risk of recurrence; but, it increases with each loss from $11 \%$ in nulliparas to $40 \%$ after three or more losses. ${ }^{9,10}$

Known risk factors for recurrent pregnancy loss are female age, previous pregnancy losses, parental structural chromosomal abnormalities, uterine abnormalities, endocrine abnormalities, antiphospholipid syndrome, and hereditary thrombophilia; even after a study, the cause is only identified in less than $50 \%,{ }^{9}$ most cases remain without a modifiable or idiopathic risk factor and within this last idiopathic factor, in recent decades the association has been reported between a state of hypercoagulability and recurrent pregnancy loss; mainly the role of reduced coagulation inhibitors in recurrent pregnancy loss of the pathogenetic role of the genetic variant associated with the hypercoagulable state in these cases ${ }^{10,11}$ There is a clear need for an evidence-based recommendation on when to initiate investigations in recurrent pregnancy loss.

Thrombophilias are alterations of the coagulation system and are classified as acquired, such is the case of antiphospholipid syndrome associated with an immune response and inherited thrombophilias, which are polymorphisms of factors that participate in the coagulation 
cascade and that generally favor the clot formation, within these factors we have the mutations, G1691A of Factor V Leiden (FVL, G1691A), G20210A of prothrombin (PRT, G20210A), G677A of methylenetetrahydrofolate reductase (MTHFR C677AT) and plasminogen activator inhibitor- 1 (4G/5G) (PAI-1, 4G/5G). ${ }^{11,12}$ Studies that report the relationship of thrombophilias with abnormalities during pregnancy, with late gestational loss, placental abruption, pre-eclampsia, fetal death, and recurrent pregnancy loss. ${ }^{13-}$ ${ }^{17}$ The objective of this study is to evaluate the relationship of FVL G1691A, PRT G20210A, MTHFR C677AT and PAI-1 4G/5G, with recurrent gestational loss and perinatal data of Mexican women who attended the Pronatal clinic in Mexico City in 2017 to $2019 .{ }^{18-21}$

\section{Material and method}

Retrospective, observational and cross-sectional study, which includes 277 pregnancies of 95 women who attended the Pronatal clinic (Hospital Bité Médica) between 2017-2019 for follow-up of pregnancy and with a study to detect hereditary thrombophilias of FVL-G1691A, PT-G20210A, MTHFR-C677T and PAI-1 4G>5G.

With the data obtained, three groups were formed:

1) Control: deliveries of patients without gestational loss, without problems during the development of pregnancy and with a study of hereditary thrombophilias,

2) Idiopathic fetal death; deliveries of patients with idiopathic gestational loss $(=1)$ and with a study of thrombophilias, and

3) Recurrent pregnancy loss; deliveries of patients with idiopathic recurrent pregnancy loss $(\geq 2)$ and with a study of hereditary thrombophilias.

The collection of age, weight and height was taken in their first consultation, by the nursing team and the collection of postnatal data was provided by the nursery area of the Bité Médica hospital, such as caesarean sections, deliveries, spontaneous abortions, weeks of gestation, weight and height of newborns.
The analysis of thrombophilias was carried out by taking a blood sample, which was sent to the laboratory of the institute of human reproductive sciences, where it was analyzed if they presented SNPs of FVL-G1691A, PT-G20210A, MTHFR-C677T and PAI-1 4G>5G, using the polymerase chain reaction (RT PCR) technique.

All patients were informed about the use and management given to their collected data (age, weight, height, postnatal data and results of thrombophilias), allowing their inclusion in this study; under informed medical consent. In addition, their anonymity is maintained, by not making reference to the origin of the information and only disclosing numerical and statistical data (as the case may be). INCLUSION CRITERIA: Women of reproductive age, with a study of thrombophilias (FVL-G1691A, PT-G20210A, MTHFR-C677T and PAI-1 4G $>5 \mathrm{G}$ ), complete records (age, weight, height, postnatal data and study of thrombophilias), with study of positive or negative thrombophilias, fetal and idiopathic death. EXCLUSION CRITERIA: Patients without a study of thrombophilias, who did not accept their inclusion in the study, systemic, genetic, neoplasms, immunological, thyroid diseases, diabetes mellitus and polycystic ovary syndrome. Variables to study: Thrombophilias, recurrent gestational loss, weeks of gestational age, weight and height of newborn.

The data of the patients were; age, weight and height and of the newborns; weeks of gestation, weight, height, are reported with mean \pm standard error (SE) and analyzed with student's test, on the other hand, thrombophilias, caesarean sections, deliveries and spontaneous abortions are reported with the percentage rate and analyzed using a chi2, in In both cases, the SPSS version 25 statistical package was used.

\section{Results}

The 95 women included in this paper did not present a significant difference in age, weight and height in the different populations evaluated, as shown in Table 1

Table I General information about the mother

\begin{tabular}{|c|c|c|c|c|c|c|c|}
\hline & $\mathbf{n}$ & Age (years) & Weight (kg) & Height (cm) & Vaginal delivery (\%) & Caesarean section (\%) & Miscarriage (\%) \\
\hline Control & 51 & $33.3 \pm 0.7$ & $67.1 \pm 1.5$ & $162 \pm 0.8$ & 50.9 & 49.01 & 0 \\
\hline PL & 61 & $32.3 \pm 0.6$ & $57.7 \pm 0.9$ & $162 \pm 0.8$ & 24.5 & 13.11 & 62 \\
\hline \multirow[t]{2}{*}{ RPL } & 155 & $33.2 \pm 0.2$ & $63.5 \pm 0.5$ & $1.63 \pm 0.5$ & 5.8 & 23.2 & 70.7 \\
\hline & & $p>0.05$ & $p>0.05$ & $p>0.05$ & - & - & - \\
\hline
\end{tabular}

Graph 1 shows the rate of each of the thrombophilias to be evaluated in the different populations, observing that FVL-G1691A only occurs in recurrent fetal loss (15.4\%) and PT-G20210A is only present in fetal death (3.2\%), meanwhile, MTHFR-C677T shows a statistically significant higher prevalence in recurrent fetal loss compared to control and fetal death $(62.1,50.9$ and $47.5 \%$, p $<0.05$ ), contrary to what happens with PAI- $14 \mathrm{G}>5 \mathrm{G}$ that presents a statistically significant lower rate in recurrent fetal loss compared to the stillbirth control (65.8 vs 82.3 and $85.2 \%, \mathrm{p}<0.05$ ).

On the other hand, in the homozygous and heterozygous versions, it was observed that FVL-G1691A only appeared in RPL with a higher prevalence in its homozygous than heterozygous form (10.3 vs 5.1\%) (Graph 1) (Graph 2), in the same way PT-G20210A it was only observed in fetal death (3.2\%) (Graph 2). As for MTHFR$\mathrm{C} 677 \mathrm{~T}$ in its homozygous form, it has a greater presence in fetal death compared to control and recurrent pregnancy loss (29.5 vs $11.7^{*}$ and $21.9 \%, \mathrm{p}<0.05$ ), different from its heterozygous form that tube lower prevalences in fetal death when compared with the control and recurrent pregnancy loss $(18.03 * *$ vs 39.2 and $43.2 \%$, $\mathrm{p}<0.05$ ). When comparing homozygous vs heterozygous of MTHFRC677T, feral death was the only population with the highest rate of homozygous compared to heterozygous (29.5 vs $18.03 \%$ ) (Graph 2). Associated with this, the highest prevalence of PAI-1 4G $>5 \mathrm{G}$ in the homozygous form was presented by the control group compared to fetal death and recurrent pregnancy loss (31.3 vs 16.3 and $24.5 \%$ ) and in the heterozygous form the highest incidence was seen in fetal death compared to control and recurrent pregnancy loss (68.8 vs 50.9 and $41.2 \% * * * . \mathrm{P}<0.05$ ), additionally, all populations present a higher prevalence of the heterozygous form in the three populations as shown in Graph 2. 


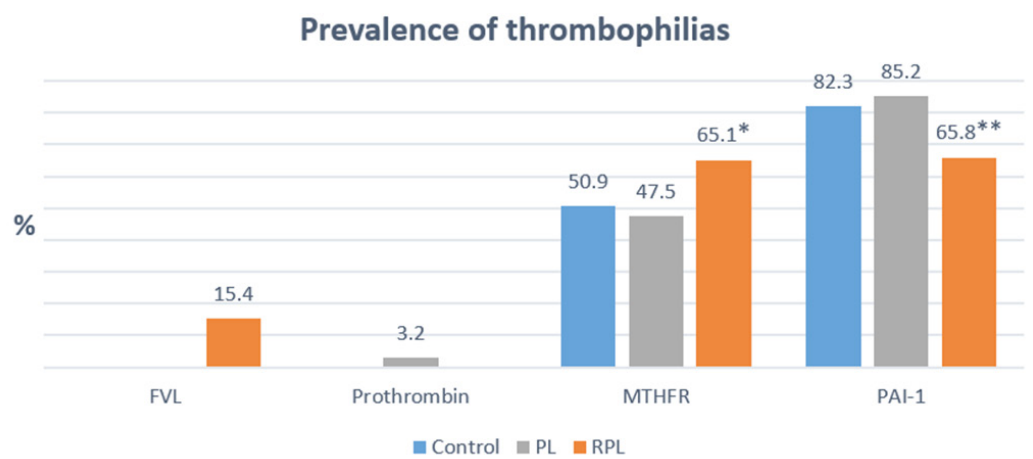

Graph I Shows the prevalence of FVL-GI69IA, PT-G20210A, MTHFR-C677T and PAI-I 4G>5G in the different populations evaluated (Control, PL and RPL). * MTHFR-C677T in RPL vs Control and PL, p<0.05, "Chi-square" and ** PAI-I 4G>5G in RPL vs Control and PL, $P<0.05$, "Chi-square".

Prevalence of thrombophilias

(Homozygous vs heterozygous)

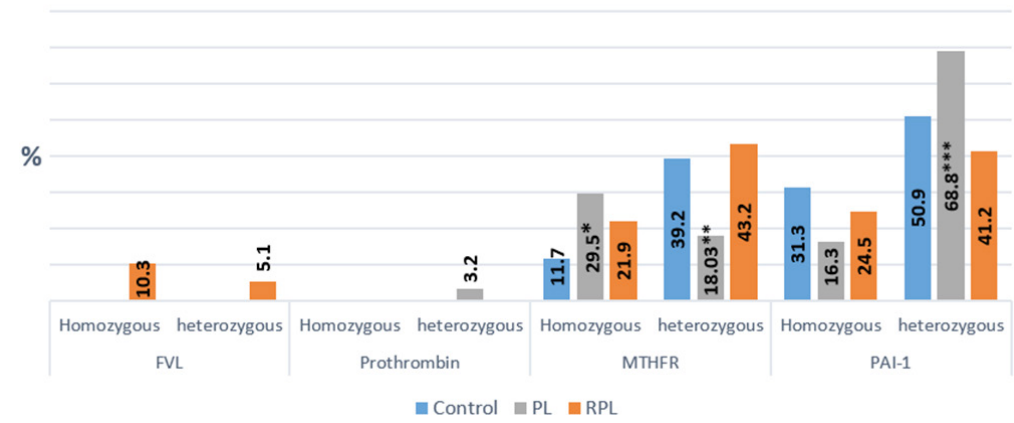

Graph 2 Shows the prevalence of FVL-GI69IA, PT-G20210A, MTHFR-C677T and PAI-I 4G>5G in their homozygous and heterozygous versions in the different populations evaluated (Control, PL and RPL). * MTHFR-C677T homozygous in control vs PL, $p<0.05$, "Chi-square", ** MTHFR-C677T heterozygous in PL vs Control and RPL, p<0.05, "Chi-square" and *** PAI-I 4G>5G heterozygous in PL vs Control and RPL, $P<0.05$, "Chi-square".

Finally, in Graph 3 we have the perinatal data which showed a decrease in the weeks of gestation of the births of mothers with recurrent pregnancy loss compared to the control and fetal death
(35.1 vs 38.8 and 39). Similarly, recurrent pregnancy loss showed a decrease in weight (2262 vs 3009.6 and 3036.1) and height (43.3 vs 48.9 and 47.2) of the newborns when compared with the control and fetal death.

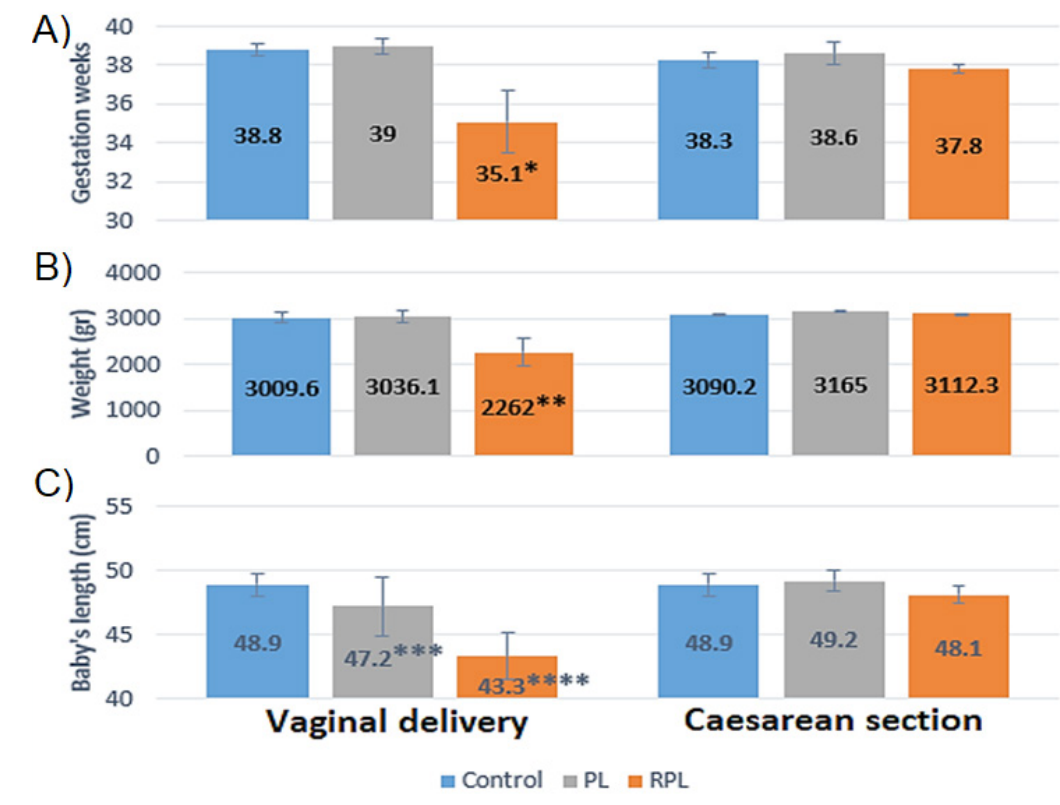

Graph 3 Shows perinatal data. A) Weeks of gestation, B) Newborn weight and D) Newborn height. * Weeks of gestation, RPL vs Control and PL, P<0.05, "Student's t", ** Weight, RPL vs Control and PL, $\mathrm{p}<0.05$, "Student's t", *** Newborn height, PL vs Control, $\mathrm{p}<0.05$, "Student's t", **** Newborn height, RPL vs Control and PL, $\mathrm{P}<0.05$, "Student's t". 


\section{Discussion}

Currently it is reported that 40 to $50 \%$ of women with recurrent pregnancy loss the cause is idiopathic; although it has been related to thrombophilias, this is still controversial. ${ }^{15,22-24}$ In Mexico there are few studies that report the incidence of thrombophilias and our research group reported that the patients presented a high prevalence of MTHFR-C677T (48.95) and PAI-1 4G> 5G (64.6\%), with a low prevalence of FVL- G1691A (3.8\%) and PT-G20210A (0.5\%). ${ }^{23}$

Therefore, the relationship between fetal death and recurrent pregnancy loss was examined with the polymorphisms of MTHFRC677T, PAI-1 4G> 5G, FVL-G1691A and PT-G20210A. This showed an increase of $14.2 \%$ ( $10.2 \%$ homozygous and $4 \%$ heterozygous) of patients with recurrent pregnancy loss who presented MTHFR-C677T, compared to the Control group (Graph 1) (Graph 2), in addition, also patients with fetal death that presented homozygous MTHFRC677T, showed an increase of $17.8 \%$, when compared with the control group (Graph 2), similarly, it was reported in 70 patients with recurrent pregnancy loss observed a $21.4 \%$ prevalence of MTHFRC677T in comparison with the control group (0\%):23 Another study ${ }^{25}$ in 245 patients with recurrent pregnancy loss observed $37 \%(30 \%$ heterozygous and 7\% homozygous) more cases with MTHFR C677T, compared to the control group; Recently ${ }^{26}$ in a meta-analysis they found that out of 10 studies analyzed, 8 presented an increase in patients with recurrent pregnancy loss who presented MTHFR C677T, where the study with the lowest incidence presented an increase of $3.7 \%$ and the one with greater than $24.5 \%(0.3$ to $17 \%$ heterozygous and 0 to $31.5 \%$ homozygous) in comparison with respective control groups (Graph 1) (Graph 2).

The explanation that PAI-1 4G>5G, appeared in $2.9 \%$ more patients with maternal death compared to the control group, reflects an increase only in the homozygous form $(17.9 \%) .{ }^{19}$ (Graph 1) (Graph 2 ), as there was an increase in patients with homozygous 4G PAI-1> $5 \mathrm{G}(12 \%)$, in 100 women with recurrent pregnancy loss, compared to the control group; Additionally, in a meta-analysis ${ }^{27}$ they report that of 18 studies, 10 had an increase in patients with recurrent pregnancy loss who presented MTHFR C677T, where the study with the lowest incidence showed an increase of $3.7 \%$ and the one with greater than $48.6 \%$ ( 2.4 to $22.4 \%$ heterozygous and 0.2 to $31 \%$ heterozygous) compared with the respective control groups (Graph 1) (Graph 2).

Unlike MTHFR-C677T and PAI-1 4G> 5G, we observed that only $15 \%$ of our patients with recurrent pregnancy loss presented FVLG1691A, as opposed to $0 \%$ in the stillbirth and control group, due to their On the other hand, the heterozygous form was presented in $10.3 \%$ and the heterozygous in $5.1 \%$, other reports their results are similar, ${ }^{28}$ with a prevalence of $4 \%$ heterozygous FVL-G1691A, which is higher than that shown by the control group $(0 \%)$, in another study of 50 patients with recurrent pregnancy $\operatorname{loss}^{29}$ analyzed, they observed an increase in patients with recurrent pregnancy loss who presented FVL-G1691A, where the minimum value found in the different studies was $3.2 \%$ and the maximum was $14.7 \%$ compared to their respective control group. In our study, PT-G20210A only appeared in $3.2 \%$ of patients with fetal death, higher than that shown by the control group and recurrent pregnancy loss $(0$ and $0 \%)$, similar to other meta-analysis reports ${ }^{22}$ where they show an increase in patients with recurrent pregnancy loss who present PT-G20210A with the lowest value presented in the analyzed studies of $0.2 \%$ and the highest of $36 \%$, with respect to the control group.

There are some studies that are associated with the presence of thrombophilias with a percentage for recurrent idiopathic pregnancy loss, but the mechanism of action is still unknown, for this reason there are studies that have analyzed placental pathologies in patients with inherited thrombophilias. finding a relationship between placental abruption and intrauterine growth restriction in patients with factor $\mathrm{V}$ Leiden mutation, which is one of the blood clotting factors. This mutation can increase the chances of developing abnormal blood clots, most often in the legs or lungs; $;^{24,27,30}$ intrauterine growth restriction, preeclampsia, and placental abruption are associated with the MTHFR mutation and in addition to these complications, gestational loss in the second trimester is associated with the PT-G20210A mutation ${ }^{31,32}$ and intrauterine growth restriction and loss gestational are associated with a PAI-1 4G mutation $>5 \mathrm{G}$; Others have found no association of thrombophilias with the increase in vascular lesions in placentas from term births and with some type of complication, but they do relate it as an underlying risk factor for lesions triggered by other processes; ${ }^{25}$ similar to the review ${ }^{26}$ where the relationship is weak in showing results of placental alterations when patients have thrombophilias, suggesting that alterations at the placental level during pregnancy is a prerequisite for thrombophilias to exert their deleterious effects. We observed a decrease in the weeks of gestational age, weight and height of newborns due to delivery in patients with a history of recurrent pregnancy loss, associated with the presence of FVL-G1691A (heterozygous and homozygous) and MTHFR C677T (heterozygous and homozygous ), with a small decrease in newborn height in patients with a history of fetal death, associated with the presence of MTHFR C677T (homozygous) and PAI-1 4G> 5G (heterozygous); which coincides with studies that report a decrease in birth weight in newborns of mothers who presented an increase in the blood of MTHFR C677T and FVL-G1691A mutations. ${ }^{27-30}$

\section{Conclusion}

The presence of inherited maternal thrombophilias increases the risk of recurrent pregnancy loss, premature delivery, lower weight and height at birth; the thrombophilias with the highest incidence that cause problems during pregnancy is MTHFR-C677T and those with low risk are FVL-G1691A, both in their homozygous or heterozygous form; studies of thrombophilias are required to identify the general trend in the Mexican population.

\section{Acknowledgments}

None.

\section{Funding}

None.

\section{Conflicts of interest}

There was no conflict of interest regarding this study.

\section{References}

1. Diejomaoh MF. Recurrent spontaneous miscarriage is still a challenging diagnostic and therapeutic quagmire. Med Princ Practice. 2015;24:38-55.

2. Barut MU, Bozkurt M, Kahraman M, et al. Thrombophilia and Recurrent Pregnancy Loss: The Enigma Continues. Med Sci Monit. 2018;24:42884294.

3. Carrington B, Sacks G, Regan L. Recurrent miscarriage: pathophysiology and outcome. Curr Opin Obstet Gynecol. 2005;17:591-597.

4. ASRM Practice Committee. Evaluation and treatment of recurrent pregnancy loss: a committee opinion. Fertil Steril. 2012;98:1103-1111. 
5. WHO. Recommended definitions, terminology and format for statistical tables related to the perinatal period and use of a new certificate for cause of perinatal deaths. Modifications recommended by FIGO as amended October 14 1976. Acta Obstet Gynecol Scand. 1977;56:247-253.

6. Practice Committee of American Society for Reproductive Medicine. Definitions of infertility and recurrent pregnancy loss. Fertil Steril. 2008;90 (5 Suppl.):S60.

7. ESHRE guideline. Recurrent pregnancy loss. ESHRE Early Pregnancy Guideline Development Group; 2017.

8. Ruth BA, Ole BC, Janine El, et al. ESHRE guideline: recurrent pregnancy loss. Human Reproduction Open. 2018:1-12.

9. Myrthe M van Dijk, Astrid M Kolte, Jacqueline L, et al. Recurrent pregnancy loss: diagnostic workup after two or three pregnancy losses? A systematic review of the literature and meta-analysis. Hum Reprod Update. 2020;26(3):356-367.

10. Maristella D’Uva, Pierpaolo Di Micco, Ida Strina, Giuseppe De Placidoa. Recurrent pregnancy loss and thrombophilia. J Clin Med Res. 2010;2(1):18-22.

11. Mateo H, Mateo S, Hernandez L, et al. Pérdida recurrente del embarazo: revisión bibliográfica. Ginecol Obstet Méx. 2016;14(8):523-534.

12. Vargas-Ruiz AG. Trombofilias hereditarias: el perfil de pruebas necesarias. Hematol Méx. 2019;20(2):79-85.

13. Estuardo LIJ, Carlos DM, Jesús APFD, et al. Incidence of hereditary thrombofilies in a population of Mexican women. Obstet Gynecol Int J. 2020;11(4):208-213.

14. Sarig J Younis, Hoffman R, Lanir N, et al. Thrombophilia is common in womenwith idiopathic pregnancy loss and isassociated with late pregnancy wastage. Fertil Seril. 2002;2(77)L342-347.

15. Barut MU, Bozkurt M, Kahraman M, et al. Thrombophilia and Recurrent Pregnancy Loss: The Enigma Continues. Med Sci Monit. 2018;24:42884294.

16. ACOG Practice Bulletin No. 197 Summary: inherited thrombophilias in pregnancy. Obstet Gynecol. 2018(132)1:249-251.

17. Masoud M, Khalili M, Soufizomorrod M, et al. Factor V Leiden 1691G $>$ A mutation andthe risk of recurrent pregnancy loss (RPL):systematic review and meta-analysis. Thrombosis J. 2020;11(18):1-16.

18. Visser J, Ulander VM, Helmerhorst FM, et al. Thromboprophylaxis for recurrent miscarriage in women with or without thrombophilia. HABENOX: a randomised multicentre trial. Thromb Haemost. 2011;105:295-301.

19. Shakarami F, Taghi M, Zare S. Association of plasminogen activator inhibitor-1 and angiotensin converting enzyme polymorphisms with recurrent pregnancy loss in Iranian women. Iran $J$ Reprod Med. 2015;13(10):627-632.
20. Chen H, Nie S, Lu M. Association between plasminogen activator inhibitor-1 gene polymorphisms and recurrent pregnancy loss: a systematic review and meta-analysis. Am J Reproduc Immunol. 2014;4(73):292-300.

21. Hamedi B, Feulefack J, Khan A, et al. Association between factor V Leiden mutation and recurrent pregnancy loss in the middle east countries: a Newcastle-Ottawa meta-analysis. Arch Gynecol Obstet. 2020;302:345354.

22. Gao H, Fang T. Prothrombin G20210A mutation is associated with recurrent pregnancy loss: A systematic review and meta-analysis update. Thrombosis Research. 2015;135:339-346.

23. Kumar A, Shrestha N, Joshi P, et al.. Association of parental methylenetetrahydrofolate reductase (MTHFR) C677T gene polymorphism in couples with unexplained recurrent pregnancy loss. BMC Research Notes. 2018;233(11):1-5.

24. Kinzler W, Prasad V, Ananth C, et al. The effect of maternal thrombophilia on placental abruption: histologic correlates. J Matern Fetal Neonatal Med. 2009;3(22):243-248.

25. Ahangari N, Doosti M, Mousavifar N, et al. Hereditary thrombophilia genetic variants in recurrent pregnancy loss. Arch Gynecol Obstet. 2018;3(300):777-782.

26. Kamli M, Hantoushzadeh S, Borna S, et al. Association between Thrombophilic Genes Polymorphisms and Recurrent Pregnancy Loss Susceptibilityin the Iranian Population: a Systematic Review and MetaAnalysis. Iranian Biomedical J. 2018;2(22):78-89.

27. Howley H, Walker M, Rodger M. A systematic review of the association between factor VLeiden or prothrombin gene variant and intrauterinegrowth restriction. Am J Obstet Gynecol. 2005;192:698-704.

28. Reddy R, Mutreja D, Moorchung N, et al. Recurrent pregnancy loss: can factor V Leiden mutationsRecurrent pregnancy loss: can factor V Leiden mutations. Obstet Gynecol Sci. 2019;62(3):179-182.

29. Sergi C, Jishi T, Walker M. Factor V Leiden mutation in women with early recurrent pregnancy loss: a meta-analysis and systematic review of the causal association. Arch Gynecol Obstet. 2020.

30. Livrinova V, Hadzi M, Hristova H, et al. Factor V Leiden, Prothrombin and MTHFR Mutation in Patients with Preeclamsia, Intrauterine Growth Restriction and Placental Abruption. Open Access Macedonian Journal of Medical Sciences. 2015;3(4):590-594.

31. Kupferminc MJ, Peri H, Zwang E, et al. High prevalence of the prothrombin gene mutation in women with intrauterine growth retardation, abruptio placentae and second trimester loss. Acta Obstet Gynecol Scand. 2000;79(11):963-967.

32. Kocher O, Cirovic C, Malynn E, et al. Obstetric complications in patients with hereditary thrombophilia identified using the $\mathrm{LCx}$ microparticle enzyme immunoassay: a controlled study of 5,000 patients. American Journal of Clinical Pathology. 2007;127(1):68-75. 